\title{
Stimulated emission from single quantum dipoles
}

\author{
Kerry $\boldsymbol{J}$ Vahala \\ Department of Applied Physics, Mail Stop 128-95, California Institute of Technology, \\ Pasadena, CA 91125, USA
}

Received 9 November 1992

\begin{abstract}
Whereas the basic features of stimulated emission are easily seen to hold true for ensembles of dipoles, the same is not always true for a single dipole system. For example, symmetry requires that well localized, bound dipoles emit a dipole field which is isotropic in the plane defined by the dipole vector. Indeed, for this case, nothing in the interaction between the dipole and the field contains information on the propagation direction of the field. These observations seem at odds with the highly directional nature of stimulated emission. We therefore test for stimulated emission by an explicit calculation considering both a single Rabi type dipole and a damped dipole whose centre-of-mass coordinates are fixed at a point. Remarkably, despite the symmetrical dipole radiation pattern, stimulated emission into the original stimulating wave is shown to occur exactly. An explanation of this effect is then considered by investigating dipoles whose centre-of-mass coordinates are allowed to vary. In this case, photon recoil of the dipole introduces a directional sense to the interaction. In the extreme opposite and trivial case of a highly delocalized dipole (centre-of-mass momentum eigenstates) stimulated emission again occurs exactly. In all intermediate cases, however, stimulated emission occurs only partially or approximately. A condition imposed on a dipole's spatial extent is established for stimulated emission to occur approximately. Finally, the original bound and localized dipole is seen to be a special limiting case of recoiling dipoles for which stimulated emission occurs exactly.
\end{abstract}

\section{Introduction}

Stimulated emission is a phenomenon of obvious importance for both fundamental and technological reasons. It is also one of the most well studied interactions that occur between an optical field and matter. In this paper we consider an issue that may, upon first examination, seem trivial and well described by the large available literature on this subject. This is stimulated emission from a single, localized, bound quantum dipole (e.g. an atomic dipole fixed in a host).

In discussing stimulated emission, a frequently quoted analytical approach considers a quantized field interacting with a two-level system [1-3]. The interaction term in the Hamiltonian is studied for the case of an initially excited system interacting with a mode containing $n_{k \sigma}$ photons ( $k$ is the wavevector and $\sigma$ is polarization). Using the nomenclature of $[1]$, Fermi's golden rule yields:

$$
\begin{aligned}
W & =\frac{2 \pi q^{2} \omega}{V \varepsilon}\left|\left\langle 1, n_{k \sigma}+1\left|a_{k \sigma} \boldsymbol{r} \cdot \boldsymbol{\sigma} \sin (\boldsymbol{k} \cdot \boldsymbol{r})\right| 2, n_{k \sigma}\right\rangle\right|^{2} \delta\left(E_{2}-E_{1}-\hbar \omega\right) \\
& =\frac{2 \pi q^{2} d_{12}^{2} \omega}{V \varepsilon}\left(n_{k \sigma}+1\right) \sin ^{2}(\boldsymbol{k} \cdot \boldsymbol{R}) \delta\left(E_{2}-E_{1}-\hbar \omega\right)
\end{aligned}
$$


where $d_{12}$ is the dipole matrix element. In deriving this result, the two-level system is assumed to have a spatial extent small in comparison to the optical wavelength so that the dipole approximation is applicable. In particular, the spatial variation of the optical wave is rcmoved from the interaction integral as $\sin (k \cdot R)$ where $R$ is the 'centre' of the two-level system. Further refinement of equation (1) would normally involve its integration with a lineshape function or a density of modes function. For the purposes of the present discussion, it is only important to note that the transition rate is proportional to $n_{k \sigma}+1$. The latter term is responsible for spontaneous decay of an excited level into vacuum. The former is the term of interest here and gives the stimulated contribution to the transition rate. In addition to showing that the transition rate itself increases with increasing photon number in a mode, this result states that emission is highly preferential: a single, well excited mode (i.e., large $n_{k \sigma}$ ) can receive the emission most of the time. This fact is central to lasing action. We will now discuss this particular aspect of quantum dipole emission in greater detail.

There are several peculiar aspects of the preferential nature of stimulated emission from bound dipoles or atoms. Consider first the process at a very intuitive level and from the 'atom's point-of-view'. Suppose that a monochromatic plane wave with wave vector $k$ is incident on a single atom and resonant with a particular two-level transition connected by a dipole matrix element. Assume further that an electron occupies the upper of the two states. Since the optical wavelength is many orders larger than the spatial extent of the atomic states, the atom sees a spatially uniform, temporally varying electric field. Indeed, in modelling the interaction, any spatial dependence of the field over the extent of the atom is strictly ignored by invoking the dipole approximation. For this reason, the direction of propagation of the field is unknown to the atom prior to emission of a photon. From this purely intuitive perspective, it therefore seems quite amazing that the atom correctly determines the appropriate direction for stimulated emission, namely, the direction of the wave vector $k$.

A semiclassical view of this situation gives an equally puzzling picture. Stimulated emission and absorption are, in fact, predicted by semiclassical analyses in which ensembles of quantum dipoles (a gas, for example) interact with a classical field ([1] pp 149-58, [4] pp. 96-110). If we consider a single quantum dipole interacting with a classical plane-wave field, however, the field radiation pattern produced by the dipole is a classical dipole field, having the axial symmetry governed by the dipole vector, but with no preferrential radiation direction in the plane defined by the dipole. An ensemble of quantum dipoles interacting with a plane wave will, however, radiate preferentially since, in effect, the dipoles form an array of radiators whose phases are such that their respective dipole emissions will constructively interfere precisely in the propagation direction of the stimulating wave. Since equation (1) obviously applies for the single dipole case, however, this would seem to be a fortuitous happenstance.

The problems associated with interpretation of stimulated emission from a single dipole, have apparently not gone unnoticed by other authors. In their text 'Laser Physics,' Sargent, Scully and Lamb note that 'these three properties-equality of phase, direction, and frequency of incident and stimulated fields-are completely obtained only for appropriately pumped media whose cross-sectional areas contain many similar dipoles [4] (p 41).' Thus, for several reasons, including the above observations based on symmetry arguments, we are first led to test for stimulated emission from an excited two-level bound dipole by explicit calculation using a classical field. Remarkably, despite a symmetrical radiation pattern resulting from a complete lack of any optical wave vector dependence in the dipole interaction, stimulated emission of one energy quantum 
is found to occur exactly into the stimulating waye. This paradox is then investigated by relaxing constraints on the dipole. We investigate dipoles that are allowed to recoil upon emission or absorption. In general, it is shown that this class of free dipoles does not exhibit perfect stimulated emission. However, for the trivial and extreme case of a totally delocalized dipole, perfect stimulated emission is recovered. This leads us to a condition imposed on dipoles if stimulated emission is to occur approximately. A special limit of this condition is seen to be the bound dipole.

\section{Stimulated emission from a single bound dipole}

The fundamental problem outlined above concerns a question of symmetry. A bound atomic dipole proned for photon emission cannot determine the propagation direction of an incident-stimulating wave. This statement is consistent with our intuition and with the very nature of the dipole approximation. We take it as our starting point in this analysis and assume that the stimulated field is a dipole radiation field (i.e. isotropic in the plane defined by the dipole vector). With this assumption, directional preference can only be brought about by the interference of this isotropic dipole field with the original stimulating wave. It is not apparent a priori, however, that this interference will lead to a reasonable result. We will therefore need to explicitly calculate the energy contained in this interference.

To clarify the essentials of the problem, we study the simplest form of stimulated emission and absorption, the classic Rabi flopping of a two-level atomic dipole. We employ a semiclassical analysis in which the two level dipole interacts resonantly with an incident plane wave of the form $E(x, t)=E_{0} \cos (k \cdot x-\omega t)$. We assume that at time $t=0$ the system is in state $|2\rangle$, the excited state, and neglect decay effects by assuming a regime in which the Rabi frequency is much faster than any decay or scattering rates. The time evolution is then given by:

$$
|\psi(t)\rangle=\exp \left(-\mathrm{i} \omega_{2} t\right) \cos \left[\left(\frac{1}{2} \Omega_{\mathrm{R}}\right) t\right]|2\rangle-i \exp \left(-\mathrm{i} \omega_{1} t\right) \sin \left[\left(\frac{1}{2} \Omega_{\mathrm{R}}\right) t\right]|1\rangle
$$

where $\omega_{1,2} \equiv \varepsilon_{1,2} / \hbar$ and where $\Omega_{\mathrm{R}} \equiv \mu_{12} E_{0} / \hbar$ is the flopping frequency. In deriving this expression, it has been assumed that the quantum states have definite parity and furthermore that their relative phases are such that $\mu_{12}$ is real (hence $\mu_{12}=\mu_{21}$ ). For a detailed derivation of this result the reader is referred to analyses in [1-4]. The atomic dipole resulting from this state vector is given by:

$$
\boldsymbol{p}=\langle\boldsymbol{\mu}\rangle=\mu_{12} \sin \left(\Omega_{\mathrm{R}} t\right) \sin (\omega t)
$$

where $\omega \equiv \omega_{2}-\omega_{1}$ is the transition frequency and also the incident field frequency by assumption. A dipole density, $\boldsymbol{P}$, for a bound dipole can be formed with this result by writing $\boldsymbol{P}=\boldsymbol{p} \delta(\boldsymbol{x})$ which, in turn, can be used as a source term in Maxwell's equations.

In the far-field (i.e. radiation zone) the field generated by this dipole is [5],

$$
E_{\mathrm{d}}(r, \theta, \phi)=\frac{k^{2}}{4 \pi \varepsilon_{0} r}(\hat{\boldsymbol{n}} \times \boldsymbol{p} \times \hat{\boldsymbol{n}}) \sin \left(k_{\mathrm{R}} r-\Omega_{\mathrm{R}} t\right) \sin (k r-\omega t)
$$

where $\hat{n}$ is a unit vector, $k=\omega / c$ is the propagation constant, $k_{\mathrm{R}} \equiv \Omega_{\mathrm{R}} / c$ and $\varepsilon_{0}$ is the dielectric constant of free space. In addition we have neglected radiation produced by the additional Rabi time dependence in the dipole. Since Rabi frequencies are typically over five orders smaller than the optical field frequency, this is a very good approximation. 
According to equation (4), the polarization wave consists of a fast optical part and a slow amplitude modulation (AM), both of which propagate outward from the dipole at speed $c$.

One reason for selecting the Rabi flopping system for this investigation stems from the fact that electron probability is precisely unity and zero at specific points in time. Therefore, there is a well defined interval of time during which a photon is emitted or absorbed. Emission cycles in this system occur for intervals of duration $T=\pi / \Omega_{\mathrm{R}}$ that begin at times $t_{\mathrm{em}}=2 m T$ for integer values of $m$. Absorption cycles occur for intervals of the same duration that begin at times $t_{\mathrm{ab}}=(2 m+1) T$. The AM wave discussed above is synchronized with these emission/absorption cycles. Therefore, at a fixed moment in time, shell-like sections of the AM wave must contain the energy added to the field by a downward flop or are less the energy removed by an upward flop. For the purposes of the calculation we refer to the shells as plus $(t)$ or minus $(-)$ shells depending on whether they were created during an emission or absorption cycle.

In the radiation zone, the total energy contained in the interference of the stimulating field and the dipole field is given by:

$$
\Delta E_{ \pm}=\varepsilon_{0} \int_{ \pm} r^{2} \mathrm{~d} r \mathrm{~d} S 2 E_{\mathrm{d}}(r, \theta, \phi) \cdot E_{0} \cos (k \cdot r-\omega t)
$$

where $\mathrm{d} S$ is the differential solid angle and the integration is performed over the entire volume defined by a plus or a minus shell. After calculation the integral in (5) reduces to the form:

$$
\Delta E_{ \pm}= \pm \hbar \omega .
$$

Thus, the stimulated photon is correctly added to the wave responsible for the stimulation, despite the fact that the atomic dipole has no indication of this wave's propagation direction.

Several comments are in order. First, the reader may wonder how computing the interference energy (equation (5)) permits the claim that the stimulated photon field has been added to the stimulating field. This is possible since equation (5) can be viewed as the inner product in function space of the two fields in question. The integral yields the projection of the dipole field onto the stimulating field multiplied by the stimulating field strength. If the dipole field projection is referred to as $\delta E_{\mathrm{d}}$, then the stimulating field amplitude is modified as $E_{0}+\delta E_{\mathrm{d}}$. To first order, the energy shift in the stimulating field is then $2 \varepsilon_{0} V E_{0} \delta E_{\mathrm{d}}$ where $V$ is the volume of a shell in question. This is just equation (5).

Second, we have computed the interference energy over a finite volume of space as necessitated by the finite duration of time during which a photon is emitted or absorbed by the dipole. For this reason, it is clear that the 'projection' of the dipole emission onto the original stimulating plane wave is only approximate with a spectral spread in $k$-space given by $k_{\mathrm{R}}$. In the limit that the stimulating wave amplitude goes to zero, however, the stimulated photon is added or removed from the stimulating wave exactly since, in this case, $k_{\mathrm{R}} \rightarrow 0$ (i.e. the flop occurs over an infinite period of time).

Third, it is interesting and important to note that although the stimulated photon is added to or removed from the stimulating field (with the provision described above), this process does not occur entirely in the direction of propagation of the stimulating wave. Indeed, it is easily shown that the total stimulated energy $(h \omega)$ is 'partitioned' equally as a pair of wavepackets in the two half spaces defined by the plane normal to 
the field propagation direction and containing the dipole. This is reasonable since, strictly speaking, the stimulating mode fills all space-so, necessarily, a portion of the dipole emission must propagate opposite to the stimulating field to completely couple to it.

The photon partitioning here is an artifact of using a semiclassical field. Although the present approach does not treat the field quantum mechanically, we can make some likely speculations as to interpretation of the above results from a quantum measurement viewpoint. Specifically, measurement of energy in one packet in a matched pair should yield a photon (or absence of a photon) half of the time that the measurement is performed. Furthermore, a correlation should exist between matched pairs of packets. Measurement of a photon in one packet requires that a corresponding measurement of the matched packet shows no photon.

Finally, a result similar to equation (6) can be readily shown using the Poynting theorem by considering the power source term $E \cdot \partial_{t} P$. However, because this source term is highly localized, this analysis only shows that net power flow across a closed surface containing the dipole includes or is less the energy $h \omega$ during the appropriately defined intervals.

To summarize the results so far: the interference of the bound dipole radiation field with the monochromatic plane wave stimulating the dipole carries the stimulated energy $h \omega$. It is possible to interpret this energy as being added to or removed from the stimulating wave with the provision that exact coupling to the true stimulating plane wave (i.e. infinite in extent) occurs only for an infinitely small stimulating field. Because the stimulating field exists over all space, the stimulated energy $h \omega$ necessarily adds to this field as an interference that propagates both with the wave and opposite to it. This fact is borne out in the calculation. Stimulated dipoles spanning a large area or contained in a large volume will reinforce this effect by providing an antenna array-like directivity. This additional directivity is not essential, however, to the basic effect. We note in appendix 2 that the case of stimulated emission from a bound and damped dipole is considered. In appendix 1 we show that certain pathologies result when a bound and local dipole interacts with multiple optical beams.

Before proceeding to the next section, we quickly consider the non-resonant case. For non-resonant excitation of the dipole, incomplete flopping of the electron probability occurs. If the electron starts initially in the upper state, then the probability of finding the electron in the lower state is given by,

$$
|\langle 1 \mid \psi(t)\rangle|^{2}=\frac{\Omega_{\mathrm{R}}^{2}}{\delta \omega^{2}+\Omega_{\mathrm{R}}^{2}} \sin ^{2}\left[\frac{1}{2}\left(\delta \omega^{2}+\Omega_{\mathrm{R}}^{2}\right)^{1 / 2} t\right]
$$

where $\delta \omega$ is the detuning frequency (i.e., difference between optical frequency and transition frequency). Proceeding as before, the energy transferred to the stimulating wave has the form

$$
\Delta E_{ \pm}=\hbar \omega \frac{\Omega_{R}^{2}}{\delta \omega^{2}+\Omega_{R}^{2}} .
$$

By comparing this to equation (7), it is clear that we could interpret the energy as that fraction of the transition energy emitted (or absorbed) during a partial flop of the electron. Since this interpretation involves partitioning a photon, we must again think in terms of a quantum measurement viewpoint. In this case, the above fraction should 
indicate the likelihood of measuring a photon added to the field during a large number of identical measurements.

Although the main result of this section, equation (6), proves that stimulated emission does occur with single localized bound dipoles, it does not provide any satisfactory physical explanation. In the next section we establish the underlying reasons for this result.

\section{Stimulated emission from a single dipole with recoil}

In allowing the centre-of-mass coordinates of a dipole to move, the possibility of dipole recoil under emission or absorption is allowed. The study of centre-of-mass momentum eigenstates of atoms interacting with coherent beams has received considerable attention owing to the importance of these states in describing the theory of laser cooling of atoms and optical manipulation of atoms [6]. For these eigenstates, stimulated emission and absorption are trivial results of the equations of motion. We therefore draw upon this approach to consider a monochromatic plane wave interacting with a dipole whose centre-of-mass wavefunction is a wavepacket. By considering the distribution of Rabi detuning frequencies in such a packet we show that the result of section 2 is a direct consequence of the more trivial centre-of-mass eigenstate result in the limit of heavy dipole wavepackets. For completeness, we also calculate the polarization density for a class of wavepackets for which stimulated emission is approximately satisfied.

We begin the analysis by writing the known time evolution of the centre-of-mass eigenstates undergoing Rabi flopping. The eigenstates of the dipole system are the set $\left\{\left|1, k_{1}\right\rangle,\left|2, k_{2}\right\rangle\right\}$ where $k_{1}$ and $k_{2}$ are the centre-of-mass momentum wave vectors for the dipole in the giound state and in the excited state, respectively. A well known result concerning the interaction of these states under resonant optical excitation is that only closed families of states interact. In the present case, only the ground and excited states for which $k_{2}-k_{1}=k$ will interact where $k$ is the optical wave vector. In deriving the equations of motion, this momentum selection rule is a direct consequence of the global nature of the interaction energy term in the Hamiltonian and the extended nature of both the atomic states and the optical plane wave. Combined with the assumption of resonant excitation, it is clear that stimulated emission into the correct optical mode is guaranteed by this selection rule. It is also clear that the absence of this same selection rule for the bound dipole in section 2 is at the root of the seemingly paradoxical result in equation (6).

The time evolution of a family of centre-of-mass momentum states undergoing resonant optical excitation is easily shown to have the form:

$\left.\left|\psi\left(k_{1}, t\right)\right\rangle=\exp \left(-\mathrm{i} \omega_{2} t\right) \cos \left[\left(\frac{1}{2} \Omega_{\mathrm{R}}\right) t\right)\right]\left|2, k_{2}=k_{1}+k\right\rangle-\mathrm{i} \exp \left(-\mathrm{i} \omega_{1} t\right) \sin \left[\left(\frac{1}{2} \Omega_{\mathrm{R}}\right) t\right]\left|1, k_{1}\right\rangle$

$$
\omega_{1,2}=\frac{1}{\hbar}\left(\varepsilon_{1,2}+\frac{\hbar^{2} k_{1,2}^{2}}{2 M}\right)
$$

where we have assumed that the system is initially in the excited state and where $M$ is the mass of the atomic system. In constructing a wavepacket using these states, it is clear that the condition of resonance cannot be satisfied simultaneously for all planewave components of the wavepacket owing to different photon recoil energies for each 
plane-wave component. As seen by the form of the solution in equation (7), states that are non-resonant will experience only partial flopping behaviour. The degree to which complete flopping occurs is determined by the magnitude of the detuning frequency $\delta \omega$ in comparison to the Rabi frequency. In each packet there will be a distribution of detuning frequencies determined by the spectral extent in $k$-space of the packet or equivalently the degree of its localization in real space. Without loss of generality the wavepacket will be defined so that its group velocity in the ground state is zero. In addition we set the optical plane wave excitation frequency $\omega$ so that:

$$
\omega=\frac{1}{h}\left(\varepsilon_{2}-\varepsilon_{1}+\frac{\hbar^{2} k^{2}}{2 M}\right)
$$

i.e., resonant with the centre of the eigenenergy distribution of the dipole transition. With this definition, the detuning frequency $\delta \omega\left(k_{1}\right)$ for a component of the wavepacket having wavevector $\boldsymbol{k}_{1}$ is given by:

$$
\delta \omega\left(k_{1}\right)=-\frac{\hbar}{M} k \cdot k_{1} .
$$

We note that equation (12) shows that in addition to the trivial plane wave case, at least two other classes of centre-of-mass solutions will exhibit exact stimulated emission. The first are solutions for which the centre-of-mass momentum is a good quantum number along the optical propagation direction but not along the other directions. A dipole sheet and a dipole wire that contain the vector $k$ are the simplest examples of this class of solution. The second class are solutions for massive dipoles. This latter class includes the situation in which a dipole is bound (infinite mass). The result of section 2 can therefore be seen as a limit in which we imagine localizing a dipole and simultaneously increasing its mass to compensate for the introduced dispersion in Rabi detuning. Stimulated emission from the resulting highly localized and bound dipole is thus a consequence of the highly delocalized interactions of each dipole plane wave component making up the dipole.

It is interesting to ask what condition is imposed on the dipole mass for approximate examples of the second class of solutions to hold true. The answer can be found in equation (7) which shows that for $\delta \omega^{2} \ll \Omega_{\mathrm{R}}^{2}$ the system undergoes approximately complete flopping. Using equation (12) this condition can be rewritten as:

$$
\frac{\hbar k}{M}\left\langle\delta k_{\|}^{2}\right\rangle^{1 / 2} \ll \Omega_{\mathrm{R}}
$$

where $\delta k_{\| l}$ is the projection of a centre-of-mass wavevector onto the optical propagation direction and $\langle>$ indicates an expectation with respect to the centre-of-mass wavepacket state. (In writing this expression we have used $\left\langle k_{1}\right\rangle=0$ according to the zero group velocity assumption.) For simple wavepackets, the root expectation of $\delta k_{\|}^{2}$ gives a measure of the wavepacket's physical extent along the direction of propagation of the optical beam and we quantify this by defining the root expectation of $\delta k_{\|}^{2}$ to be $2 \pi / \lambda$ where $\lambda$ is a measure of the wavepacket's physical extent. For example, $\lambda$ would be proportional to the RMS variance of the wavepacket in real space. With this definition, condition (13) can be rewritten as:

$$
\frac{\hbar k}{M} T_{\mathrm{R}}=v_{\mathrm{r}} T_{\mathrm{R}} \ll \lambda
$$


where $T_{\mathrm{R}}$ is the Rabi period and $v_{\mathrm{r}}=h k / M$ is the dipole recoil velocity. In this form the validity condition states that the mass of a dipole is correct in proportion to its physical extent provided that the dipole does not recoil by a distance comparable to its own physical extent along the direction of optical propagation during one Rabi period. This condition also points out that stimulated emission will occur for Rabi periods that are made sufficiently short (or equivalently excitation fields sufficiently intense) to guarantee minimal spatial recoil.

It is important to note that although the condition $\delta \omega^{2} \ll \Omega_{\mathbb{R}}^{2}$ does guarantee complete flopping it does not eliminate all dependence of the state vector evolution on detuning. It is easily shown that when this condition holds the time evolution of the ground and excited state amplitudes are modified by a phase factor $\exp \left( \pm \frac{1}{2} \mathrm{i} \delta \omega t\right)$, respectively. This phase term amounts to an energy shift in the levels caused by the optical interaction. In the present case, its form results from photon recoil and is given by $-\hbar k \cdot k_{1} / 2 M$. It therefore alters the energy-momentum dispersion relations for both the excited and the ground states. This modification is distinct from that already present in the excited state due to the absorbed photon momentum and partially offsets it. To understand the effect of this term we will compute the time evolution of the dipole density. The time evolution of the dipole wavepacket state vector is given by:

$$
\begin{aligned}
|\psi(t)\rangle=\exp \left(-\mathrm{i} \varepsilon_{1} t / \hbar\right)[ & \exp (-\mathrm{i} \omega t) \cos \left(\frac{1}{2} \Omega_{\mathrm{R}} t\right)|2\rangle \int \mathrm{d} k_{1} a\left(k_{1}\right) \exp \left(-\frac{1}{2} \mathrm{i} \delta \omega\left(k_{1}\right) t\right) \\
& \times \exp \left(-\frac{1}{2} \mathrm{i} \hbar k_{1}^{2} t / M\right)\left|k_{1}+k\right\rangle-\mathrm{i} \sin \left(\frac{1}{2} \Omega_{\mathrm{R}} t\right)|1\rangle \int \mathrm{d} k_{1} a\left(k_{1}\right) \\
& \left.\times \exp \left(-\frac{1}{2} \mathrm{i} \delta \omega\left(k_{1}\right) t\right) \exp \left(-\frac{1}{2} \mathrm{i} i k_{1}^{2} t / M\right)\left|k_{1}\right\rangle\right]
\end{aligned}
$$

where the ground state wavepacket state (centre-of-mass coordinates only) is given by:

$$
|F(t)\rangle=\int \mathrm{d} k_{1} a\left(k_{1}\right) \exp \left(-\frac{1}{2} \mathrm{i} h k_{1}^{2} t / M\right)\left|k_{1}\right\rangle .
$$

Using equation (15) it is straightforward to compute the expectation of the dipole density operator $\boldsymbol{P}=\boldsymbol{\mu}|\boldsymbol{x}\rangle\langle\boldsymbol{x}|$. The result is:

$$
\langle\boldsymbol{P}\rangle=-\left|F\left(\boldsymbol{x}-\frac{\hbar k}{2 M} t, t\right)\right|^{2} \mu_{12} \sin \left(\Omega_{\mathrm{R}} t\right) \sin (k \cdot x-\omega t)
$$

where $F(x, t) \equiv\langle x \mid F(t)\rangle$ is the ground state wavefunction for the wavepacket without any optical interaction.

The dipole density is therefore a plane wave in quadrature with the original stimulating field. It resides in a region of space defined by the probability density of the dipole wavepacket which itself is in motion with group velocity $\hbar k / 2 M$. This motion is at half of the recoil velocity. It shows that both the ground state and excited state wavepackets move in unison (this can also be seen in equation (15)). This is somewhat counter-intuitive since, by assumption, the ground state has zero group velocity and the excited state group velocity is the recoil velocity. Indeed, if this situation persisted, the wavepackets for each state should drift apart leading to eventual non-overlap and shut-off of stimulated emission. This does not occur, however, owing to the phase terms discussed above. Their effect is to impart half of the recoil momentum in the ground state wavepacket while removing the same amount from the excited state wavepacket, thus balancing the group velocities of both wavepackets. Although the motion of the 
dipole packet at half recoil velocity may seem at first strange, it does agree with our notion of the dipole's speed over several absorption-emission cycles in which, on average, only half of the time does the dipole reside in the upper state and advance with the full recoil velocity.

Finally, let us consider again the issue of stimulated emission from the dipole density calculated in equation (17). Although we have explained why stimulated emission occurs in the important case of interest and for several additional cases, it is illustrative to revisit the calculation except now using a centre-of-mass state which is plane-wave like. In the present context this is a wavepacket which extends in all directions for distances that are large in comparison to the optical wavelength. Optical power launched by such a dipole will efficiently couple to the stimulating wave due to the form of equation (17). Therefore, in this special case, rather than computing the radiated power as was done in section 2 , it is possible to compute the energy directly added to or removed from the stimulating wave in the vicinity of the dipole wave. By starting with the Poynting theorem, we integrate over all space and over one Rabi downward or upward flop ( \pm as in section 2) to conclude that:

$$
\int_{ \pm} \mathrm{d} t \mathrm{~d} x \boldsymbol{E} \cdot \partial_{r} P= \pm \hbar \omega
$$

\section{Conclusion}

The main results of this investigation have been to show first, that stimulated emission occurs in highly localized and bound single quantum dipole systems. Stimulated quanta are correctly added to (or removed from) the stimulating plane wave by the dipole, despite a total lack of information concerning the vector nature of $k$ (the wavevector of the plane wave) in the dipole-field interaction. Unlike a conception of stimulated emission in which the stimulated wave is launched in the same direction as the stimulating wave (as would happen in a volume array or gas of dipoles), a single dipole radiates isotropically in the plane defined by the dipole vector. The projection of this isotropic field onto the original stimulating wave has been shown to contain the stimulated energy. This energy flows into the stimulating plane wave in both the direction of propagation and opposite to this direction. Coupling to the stimulated wave is perfect only for the case of an infinitely long Rabi flop. Second, we have explained the above effect as a direct consequence of a more straightforward result for centre-ofmass momentum eigenstates of quantized dipoles. We established the bound dipole result as a limit in which a dipole wavepacket is increasingly localized while increasing its mass to compensate for momentum recoil dispersion. In addition we established a condition on dipole wavepacket extent and mass in order for stimulated emission to occur. The motion of a dipole density wavepacket satisfying this condition was solved and the packet was shown to advance under resonant excitation with a group velocity that is exactly half of the recoil velocity.

\section{Appendix 1. Interaction with two plane waves}

Some interesting pathologies are possible as a result of a bound dipole's highly local view of the interaction. We briefly consider an example in which two plane waves of 
equal frequency but differing propagation directions are incident on the dipole. For convenience we also assume that the waves have identical linear polarization states. The forms assumed are:

$$
\begin{aligned}
& E_{1}=\hat{\boldsymbol{e}}_{z} E_{01} \cos \left(k_{1} r-\omega t\right) \\
& E_{2}=\hat{\boldsymbol{e}}_{z} E_{02} \cos \left(\boldsymbol{k}_{2} r-\omega t-\phi\right) .
\end{aligned}
$$

Proceeding as before, the polarization of the dipole is given by:

$$
p=\mu_{21} \sin \left(k_{\mathrm{R}} r-\Omega_{\mathrm{R}} t\right) \sin (\omega t+\psi)
$$

where the $\psi$ and $\Omega_{\mathrm{R}}$ are given by:

$$
\begin{aligned}
& \mathrm{e}^{\mathrm{i} \psi}=\frac{E_{01}+E_{02} \mathrm{e}^{\mathrm{i} \phi}}{\left|E_{01}+E_{02} \mathrm{e}^{\mathrm{i} \phi}\right|} \\
& \Omega_{\mathrm{R}}=\frac{\mu_{12}}{\hbar}\left|E_{01}+E_{02} \mathrm{e}^{\mathrm{i} \phi}\right| .
\end{aligned}
$$

The energies transferred to each mode in plus and minus shells take the form:

$$
\begin{aligned}
& \Delta E_{1}= \pm \hbar \omega \frac{E_{01}\left(E_{01}+E_{02} \cos \phi\right)}{E_{01}^{2}+E_{02}^{2}+2 E_{01} E_{02} \cos \phi} \\
& \Delta E_{2}= \pm \hbar \omega \frac{E_{02}\left(E_{02}+E_{01} \cos \phi\right)}{E_{01}^{2}+E_{02}^{2}+2 E_{01} E_{02} \cos \phi} .
\end{aligned}
$$

Obviously, we have $\Delta E_{1}+\Delta E_{2}= \pm \hbar \omega$ indicating that as before a single photon of energy is added or removed by the dipole from the combined energies of the fields during one flopping cycle. The 'partitioning' of this photon in the semiclassical analysis depends on the relative phases of the plane waves. For in-phase waves (i.e. $\phi=0$ ) we have:

$$
\Delta E_{j}= \pm \hbar \omega \frac{E_{0 j}}{E_{01}+E_{02}}
$$

indicating a partitioning according to the relative field amplitudes $(j=1,2)$. For out-of-phase plane waves $\left(\phi=\frac{1}{2} \pi\right)$ we find:

$$
\Delta E_{j}= \pm \hbar \omega \frac{E_{0 j}^{2}}{E_{01}^{2}+E_{02}^{2}}
$$

so that partitioning according to plane wave power occurs. For both of these cases, symmetrical partitioning occurs when $E_{01}=E_{02}$. As for the detuned discussion, one should think in terms of a quantum measurement on each mode performed during one cycle of the Rabi flop. The above ratios can then be viewed as the fraction of the time over a series of measurements that the emitted (or absorbed) photon is found in a particular mode.

A more peculiar effect occurs when the plane waves are out-of-phase $(\phi=\pi)$. For 
this case we find:

$$
\begin{aligned}
& \Delta E_{1}= \pm \hbar \omega \frac{E_{01}}{E_{01}-E_{02}} \\
& \Delta E_{2}=\mp \hbar \omega \frac{E_{02}}{E_{01}-E_{02}} .
\end{aligned}
$$

During a given cycle, each wave can lose or gain more than one photon. Since the atomic dipole can supply only one photon, this additional energy actually represents a transferrence of energy from one mode to the other. It should be noted that the singularity in these expressions is an artifact of integration over an interval defined by the Rabi period. This period becomes infinite (no flopping occurs) for the case $E_{01}=E_{02}$ with the phase angle $\phi=\pi$.

\section{Appendix 2. Stimulated emission from damped dipoles}

The more familiar form of stimulated emission occurs from dipoles so strongly damped that Rabi flopping does not occur. Although precise identification of quantum emission and absorption is impossible in these systems, we include an average power analysis for completeness. In two-level systems damping is frequently described phenomenologically using the population relaxation time constant $T_{2}$ within the density matrix formalism $[1,4]$. We must hypothesize that the dipole studied here is interacting with a bath in addition to the stimulating wave. The bath interactions are responsible for the damping and, for the case of an inverted system, also allow pumping of the transition. By applying such an approach, it is straightforward to show that the induced dipole is given by:

$$
p=\frac{\left|\mu_{12}\right|^{2}}{\hbar}\left(n_{2}-n_{1}\right) E_{0}\left(\frac{(\Omega-\omega) \cos (\omega t)+\gamma \sin (\omega t)}{(\Omega-\omega)^{2}+\gamma^{2}}\right)
$$

where $n_{2}-n_{1}$ is the population inversion, $\gamma \equiv 1 / T_{2}$, and where the rate equation approximation has been invoked ([4] p 203). Also note that we allow for non-resonant processes here since $\Omega$, the transition frequency, is distinct from the plane wave frequency $\omega$. The form of the incident plane wave is assumed to be identical to before. Of the two dipole time dependences appearing in equation (A2.1), both scatter energy, but only the $\sin (\omega t)$ carries away dipole power by successfully interfering with the stimulating wave in the far field. This interference is computed as before yielding the stimulated power,

$$
P_{\mathrm{s}}=\frac{\omega\left|\mu_{12}\right|^{2}}{2 \hbar} \frac{\left(n_{2}-n_{1}\right) \gamma E_{0}^{2}}{(\Omega-\omega)^{2}+\gamma^{2}}
$$

Stimulated emission depletes the population inversion $n_{2}-n_{1}$. The depletion rate is given by the expression:

$$
\frac{\mathrm{d}\left(n_{2}-n_{1}\right)}{\mathrm{d} t}=-\frac{\left|\mu_{12}\right|^{2}}{\hbar^{2}} \frac{\left(n_{2}-n_{1}\right) \gamma E_{0}^{2}}{(\Omega-\omega)^{2}+\gamma^{2}} .
$$


The power loss (or gain) by the transition is then $P_{\mathrm{T}}$ where:

$$
P_{\mathrm{r}}=\frac{1}{2} \hbar \Omega \frac{\mathrm{d}\left(n_{2}-n_{1}\right)}{\mathrm{d} t} .
$$

For resonant excitation $(\Omega=\omega)$ it is apparent that $P_{\mathrm{s}}=-P_{\mathrm{T}}$ so that on the average, the power lost by the dipole is gained by the stimulating plane wave. For non-resonant excitation, however, there is a deficit or surplus in the far-field power. This difference we call $\Delta P$ where:

$$
\Delta P \equiv P_{\mathrm{s}}-P_{\mathrm{T}}=(\omega-\Omega) \frac{\left|\mu_{12}\right|^{2}}{2 \hbar} \frac{\left(n_{2}-n_{1}\right) \gamma E_{0}^{2}}{(\Omega-\omega)^{2}+\gamma^{2}} .
$$

This interesting effect which did not occur in the Rabi case, is possible because of bath damping of the dipole. The deficit or surplus power is provided by the bath. For $\triangle P>0$ energy is transferred from the bath (the bath is cooled by the stimulating field) and for $\Delta P<0$ energy is transferred into the bath (the bath is heated).

\section{References}

[1] Yariv A 1975 Quantum Electronics 2nd edn (New York: Wiley) p 159

[2] Sakurai J J 1984 Advanced Quantum Mechanics (Menlo Park, CA: Benjamin/Cummings) pp 36-47

[3] Townsend J S 1992 A Modern Approach to Quantum Mechanics (New York: McGraw-Hill) p 431

[4] Sargent M III, Scully M and Lamb W Jr 1974 Laser Physics (Reading, MA: Addison-Wesley)

[5] Jackson J D 1975 Classical Electrodynamics 2nd edn (New York: Wiley) p 395

[6] J. Opt. Soc. Am 1985 B 2 (11); 19896 (11). Special issues 